\title{
The DAO Liquid Crystal Spectropolarimeter dimaPol
}

\section{Dmitry Monin, David Bohlender, Tim Hardy and Les Saddlemyer}

Dominion Astrophysical Observatory, Herzberg Astronomy and Astrophysics Program, National Research Council of Canada, 5071 West Saanich Road, Victoria, BC V9E 2E7, Canada

email: Dmitry.Monin@nrc-cnrc.gc.ca

\begin{abstract}
The spectropolarimeter dimaPol measures circular polarization in spectral lines of stellar objects. The instrument is used to simultaneously detect polarization signals in the hydrogen $\mathrm{H} \beta$ line as well as nearby metallic lines. A fast switching ferro-electric liquid crystal waveplate synchronized with charge shuffling on the CCD is employed to greatly reduce instrumental systematics. dimaPol has been in use on the DAO 1.8-m Plaskett telescope since 2007. In this presentation we show the capabilities of the instrument as well as some of the main results obtained with it to date.
\end{abstract}

Keywords. polarization, magnetic fields, instrumentation: polarimeters, stars: magnetic fields

\section{What is dimaPol?}

dimaPol is an inexpensive plug-in module for the Cassegrain spectrograph on the DAO $1.8-\mathrm{m}$ Plaskett telescope. It is employed to perform medium resolution $(R \approx 10,000)$ circular spectropolarimetry of stars in an approximately $280 \AA$ wide wavelength region centered on the $\mathrm{H} \beta$ line. The instrument is used to study global magnetic fields in stars through the Zeeman effect. In the presence of a magnetic field spectral lines split into groups of components with opposite circular polarization. The amount of splitting is proportional to the longitudinal field strength.

dimaPol has some important advantages: it allows one the simultaneous detection of polarization signals in the hydrogen $\mathrm{H} \beta$ as well as metallic lines; stars in a wide range of rotational velocities can be studied because the contrast in hydrogen lines, and the amplitude of the associated polarization signal, is less affected by rotational broadening; a higher polarimetric precision can be reached thanks to instrument's fast switching capabilities.

The module consists of a fixed quarter-wave plate, a switchable half-wave plate, and a calcite beam displacer. There are no moving parts which greatly simplifies the design. The quarter-wave plate converts the two circularly polarized beams into two orthogonal, linearly polarized beams. The beams are then separated by the beam displacer. The switchable half-wave plate is a ferroelectric liquid crystal (FLC); the FLC is a bi-stable electrically switchable device. A voltage applied to it rotates linearly polarized light by $90^{\circ}$. As a result, the two beams exchange places on the detector as the voltage is switched. The charge on the CCD is shuffled back and forth in sync with the switching FLC plate. This helps to minimize most instrumental effects such as seeing effects, guiding errors, the slightly different optical paths of the two beams, the spectrograph response, pixel-topixel CCD sensitivity variations, etc. The switching can be done at rates up to $100 \mathrm{~Hz}$. The accuracy of polarization measurements is improved by a factor of 2 or more when more than 30 switch cycles are executed (for details see Monin et al. 2012). FLC plates 

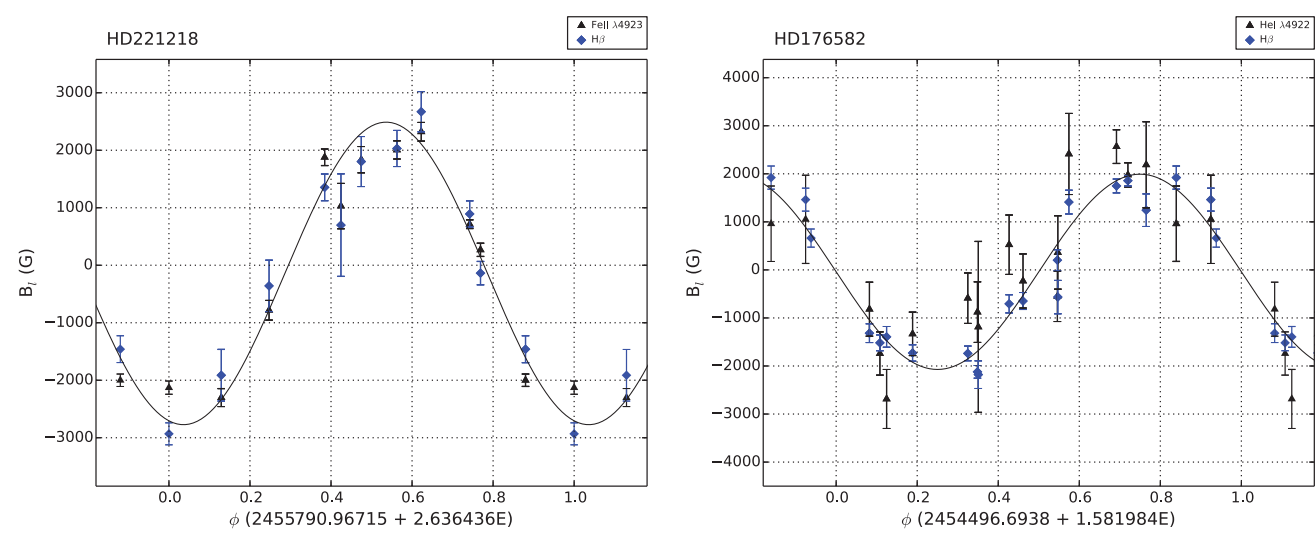

Figure 1. Longitudinal magnetic field curves: HD 221218 and HD 176582 - two of nine new magnetic B-type stars discovered with dimaPol.

have been used in solar polarimeters before but as far as we know dimaPol is the first night time polarimeter to use such a plate.

\section{Ongoing projects using dimaPol}

dimaPol is scheduled for a significant amount of time on the DAO 1.8-m Plaskett telescope. The instrument is very easy to use. Measuring the magnetic shift happens in nearly real time. The result is known before the telescope is moved to the next target! 944 observations of $137 \mathrm{O}-\mathrm{F}$ type stars have been obtained so far. Most of these stars were observed as part of the Dominion Astrophysical Observatory Magnetic Field Survey. Some of the results of this survey as well as the results from our two other ongoing projects that use dimaPol are outlined below.

\subsection{The Dominion Astrophysical Observatory Magnetic Field Survey (DMFS)}

An extensive survey of upper main sequence stars is being carried out with the DAO 1.8-m telescope and dimaPol. By including mid-B to A-type stars the survey serves as an extension to the Magnetism in Massive Stars (MiMeS) CFHT Large Program. The data for more than 100 stars, including known magnetic and null standards, have been obtained. Nine new magnetic B-type stars have been discovered so far in the course of the survey. Figure 1 shows the longitudinal magnetic field curves for two of these nine new magnetic stars. For six of these we have found periods. We have also found or improved rotation periods of a number of previously known magnetic stars. Our accuracy in $\mathrm{H} \beta$ is often better than what was obtained in the past with other instruments.

\subsection{Hydrogen Versus Metallic Magnetic Field Measurements}

Some of the magnetic stars show a discrepancy between the field strength derived from hydrogen and metallic lines. This has been known for a long time and is usually attributed to the inhomogeneous distribution of metals over the stars surfaces. But it has been unclear what part of it is due to the instrumentation used and/or data reduction methods. Magnetic fields in hydrogen lines and metallic lines are usually measured with different instruments. We have looked at a number of known magnetic stars with dimaPol and found that some of the discrepancy is instrumental in nature and can be corrected. Photoelectric measurements in the wings of hydrogen lines reported in the past need to be corrected for the Stark effect in order to agree with our line core measurements 

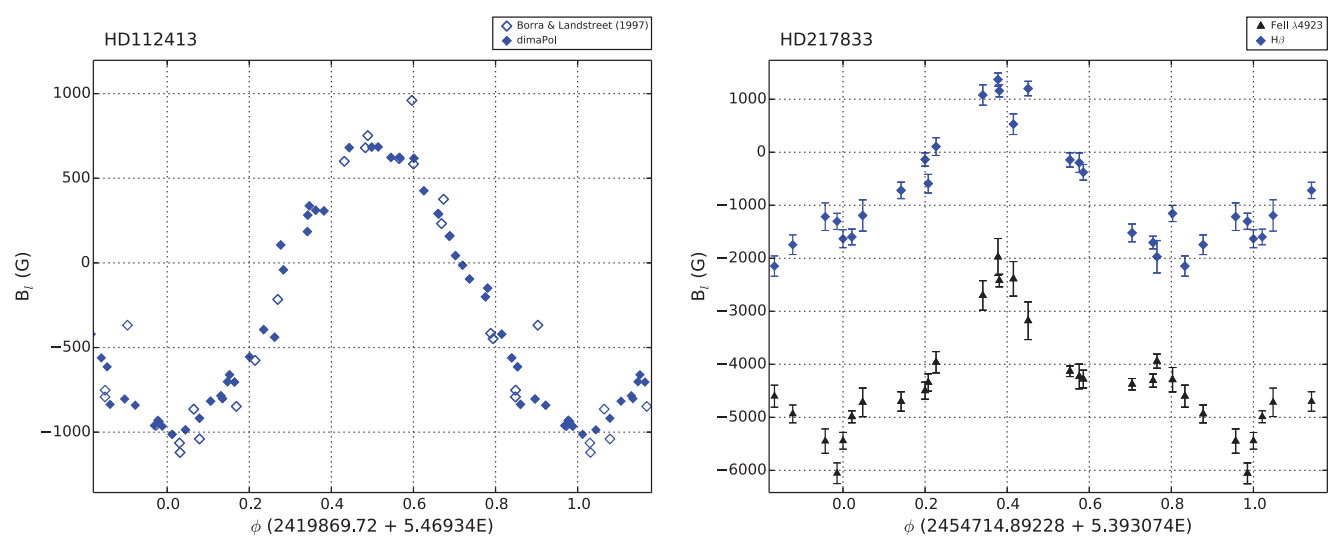

Figure 2. Longitudinal magnetic field curves: HD 112413 - correction for the Stark effect makes the photoelectric $\mathrm{H} \beta$ measurements by Borra \& Landstreet (1997) agree with our higher accuracy line core measurements. Note that thanks to the long baseline we were able to improve the period in this well known magnetic star; HD 217833 - a huge discrepancy between hydrogen and iron. The dipole field strength derived from metallic lines only can be overestimated by a factor of 4 ! Hydrogen magnetic measurements are crucial for understanding the field geometry.

(see Figure 2). This is in good agreement with the theoretical work by Mathys et al. (2000). In stars in which real discrepancies exist the magnetic field geometry can be derived incorrectly if only metallic lines are used. HD 217833 is an extreme example where the polar magnetic field derived in the past (Elkin 1994) using metallic lines was overestimated by a factor of 4 ! Thanks to dimaPol we were also able to establish that the dipole in this star, assuming the field is predominantly dipolar, is likely tilted by almost $90^{\circ}$ from the rotational axis. Before it was believed to be almost parallel. This example emphasizes the importance of obtaining hydrogen line polarimetric observations when attempting to reconstruct the field geometry.

\subsection{Searching for Magnetic Fields in B-type Stars in the Pleiades.}

Landstreet et al. (2008) studied magnetic stars in open clusters and found that the magnetic field decays rather rapidly during the main sequence stage. Strong $\mathrm{kG}$ fields are only observed near the ZAMS. The field decays faster in more massive stars. Large fields in 4-5 solar mass stars disappear on a time scale of 15 Myrs. Silvester et al. (2006) reported a detection of a kG magnetic field in a Pleiades member: HD 23850 (B8III, 4.5 solar masses). This is quite interesting as the Pleiades open cluster is 100 Myrs old. Strong magnetic fields in B-type stars are expected to decay significantly in a cluster of that age. We have observed HD 23850 with dimaPol nine times. No magnetic field was detected. We are now surveying 16 other B-type stars in the cluster. Most of them are fast rotators and are difficult targets for most existing polarimeters but dimaPol is well suited to the task. We have observed nine stars so far, many of them multiple times. A typical error bar is $0.1 \mathrm{kG}$ and so far no fields have been detected. This is in agreement with the results of Landstreet et al. (2008).

\section{References}

Borra, E. F. \& Landstreet, J. D. 1997, ApJ 212, 141

Elkin G. V. 1994, in: J. Zverko, and J. Ziznovsky (eds.), Chemically peculiar and magnetic stars (Tatranska Lomnica, Slovak Republic) p. 35 
Landstreet, J. D., Silaj, J., Andretta, V., Bagnulo, S., Berdyugina, S. V., Donati, J.-F., Fossati, L., Petit, P., Silvester, J., \& Wade, G. A. 2008, A\&3A 481, 465

Mathys, G., Stehlé, C., Brillant, S., \& Lanz, T. 2000, A $\& A$ 358, 1151

Monin, D., Bohlender, D., Hardy, T., Saddlemyer, L., \& Fletcher, M. 2012, PASP 124, 329

Silvester, J., Wade, G. A., \& Hanes, D. A. 2006, in: R. Casini \& B. W. Lites (eds.), Solar Polarization 4, ASP Conf. Series 358 (San Francisco: ASP), p. 409 